\title{
Optical coherence tomography angiography and the visual field in hypertensive and normotensive glaucoma
}

\author{
Marketa Zakovaa , Jan Lestak ${ }^{\mathrm{a}, \mathrm{b}}$, Martin Fus ${ }^{\mathrm{a}, \mathrm{b}}$, Klara Maresovac
}

\begin{abstract}
Background. Hypertensive glaucoma (HTG) causes damage to the retinal ganglion cells and eventually to the entire visual pathway due to high intraocular pressure (IOP). However, increased IOP will also affect the vessel density (VD) of the posterior pole of the eye and the related retinal ganglion nerve fibres (RNFL). In normotensive glaucoma (NTG), the retinal ganglion cells are relatively intact. The pathology is at the level of ganglion fibres. The unanswered question is what has altered ganglion cell fibres at the level of the retina and optic nerve head in NTG?

Aim. The aim of this study was to determine whether there is a correlation between the retinal nerve fibre layer (RNFL) and vessel density (VD) at the same altitudinal half of the retina and the sum of sensitivities of the contralateral half of the visual field of the same eye in hypertensive and normotensive glaucoma (NTG).

Methods. Our group included 20 patients with HTG and 20 patients with NTG. The Pearson's correlation coefficient $r$ was used for evaluation of the relationship of the peripapillary RNFL and VD, visual field (using the fast threshold glaucoma program) as the sum of sensitivities in apostilbs (asb) to the extent of 0-22 degrees. The results of sensitivity were compared with the RNFL and VD of the contralateral altitudinal half of the retina in the same eye.

Results. In the HTG group there was a moderate relationship between RNFL and VD (both hemifields), but no relationship between RNFL and VF. VD SH and VF IH showed weak correlation and VD IH and VF SH showed no correlation. In patients with NTG, we found a strong correlation between RNFL and VD (both hemifields), between VD SH and VF IH a moderate correlation, between VD IH and VF SH also a moderate correlation and a weak correlation between RNFL and VF.

Conclusion. By comparing the RNFL and VD at the same altitudinal halves of the retina, we found a moderate correlation in HTG and a strong correlation in NTG. We found no or a weak correlation between VD and VF in HTG. In NTG the relationship between VD and VF showed a strong correlation. These findings reveal the differences in the diagnostic groups.
\end{abstract}

Key words: retinal nerve fibre layer, optical coherence tomography angiography, vessel density, visual field, hypertensive glaucoma, normotensive glaucoma

Received: September 23, 2020; Accepted: September 23, 2020; Available online: October 15, 2020

https://doi.org/10.5507/bp.2020.044

(c) 2021 The Authors; https://creativecommons.org/licenses/by/4.0/

${ }^{a}$ CTU in Prague, Faculty of Biomedical Engineering, 27201 Kladno 2, Czech Republic

${ }^{b}$ Eye clinic JL Faculty of Biomedical Engineering CTU in Prague, 15800 Prague 5, Czech Republic

'Department of Ophthalmology, Faculty of Medicine and Dentistry, Palacky University Olomouc, Czech Republic

Corresponding author: Jan Lestak, e-mail: lestak@seznam.cz

\section{INTRODUCTION}

Hypertensive glaucoma (HTG) causes damage to the retinal ganglion cells and consequently to the entire visual pathway due to high intraocular pressure (IOP) $\left(\right.$ ref. $\left.^{1}\right)$. However, increased IOP will also affect the vessel density (VD) of the posterior pole of the eye and the related retinal ganglion nerve fibres (RNFL) $\left(\right.$ ref. $\left.^{2-4}\right)$. In normotensive glaucoma (NTG) the retinal ganglion cells are relatively intact. The pathology is in the level of ganglion fibres. The unanswered question is what has altered ganglion cell fibres at the level of the retina and optic nerve head in NTG? OCTA is a new imaging modality that has begun to expand our knowledge of the role of ocular blood flow in glaucoma?

Peripapillary perfusion parameters are better than macular perfusion parameters for glaucoma diagnosis, supporting the idea that glaucomatous superficial retinal vascular changes are more pronounced in the peripapillary region ${ }^{8}$.

Therefore, in this investigation we focused on evaluation of peripapillary VD. Our previous work showed a greater correlation of the VD of small vessels (VDs) than of all vessels (VDa) (ref. ${ }^{9}$ ). The results of previous work also gave us some clues, where we examined the progression of changes in the visual fields in HTG (treated by prostaglandins and beta-blockers) and NTG for five years with or without various types of topical treatment (prostaglandins and beta-blockers). We found that in eyes where prostaglandins were used that affected the posterior pole vessels of the eye or the eye was untreated, there was a statistically significant progression of changes in visual fields, especially in NTG.

This means that the vessels of the anterior part of the optic nerve may play an important role in the pathogenesis of NTG. Therefore, the aim of this work was to 
determine whether in the HTG and NTG group there is a correlation between RNFL, vessel density in the peripapillary region (VD) in the same altitudinal half of the retina to the sum of sensitivities of the bilateral half of the visual field of the same eye.

\section{PATIENTS AND METHODS}

Our group included 20 patients with HTG, 13 females of mean age 64.7 years (54-73 years) and 7 males mean age 60.8 years (53-73 years) and 20 patients with NTG: 17 females of mean age 56.1 years (43-79 years) and 3 males of mean age 60 years (51-66 years). Inclusion criteria: visual acuity 1.0 with possible correction of less than \pm 3 dioptres, approximately the same changes in visual field in all patients, whereas initial HTG and NTG were concerned without any other ocular and neurological disease. Complex ophthalmology examinations including electrophysiological examination were performed in all patients to confirm glaucoma. The RNFL and peripapillary VD were measured peripapillary, using Avanti RTVue $\mathrm{XR}$ from Optovue. Visual field was examined using the fast threshold glaucoma program in the Medmont M-700 machine (Medmont International Pty Ltd Australia). The sum of sensitivities in apostilbs (asb) was evaluated in the extent of 0-22 degrees both in the upper and lower half of the visual field. The results of sensitivity were compared with the RNFL and VD of the contralateral altitudinal half of the retina in the same eye. Each group (HTG and NTG) was evaluated separately. The Pearson's correlation coefficient $r$ was used for evaluation of the relationship of the selected parameters.

\section{RESULTS}

Measured values of RNFL, VD and visual field in HTG and NTG groups are shown in Tables 1 and 2. Pearson's correlation coefficients for both groups are shown in Table 3.

The Pearson's correlation coefficient $(P=0.001)$ was used for evaluation of the relationship of the selected parameters. By comparing the RNFL and VD in HTG in the upper halves of the retina $(\mathrm{r}=0.5)$ and in the lower halves of the retina $(\mathrm{r}=0.51)$, we found a moderate correlation. By comparing the VD from the upper half of the retina and the contralateral sum of sensitivities in the visual field $(r=0.2)$, we found a weak correlation. There was no correlation between VD from the lower half of the retina and the sum of sensitivities in the upper half of the visual fields $(r=0.04)$. We found even less correlation between RNFL SH and VF IH ( $\mathrm{r}=-0.04)$ and between RNFL IH and VF SH ( $\mathrm{r}=-0.12)$.

By comparing the RNFL and VD in NTG in the upper halves of the retina $(r=0.62)$ and in the lower halves of the retina $(r=0.6)$, we found a strong correlation. By comparing the VD from the upper half of the retina and the contralateral sum of sensitivities in the visual field $(\mathrm{r}=0.7)$, we also found a strong correlation. We found a moderate relationship $(\mathrm{r}=0.52)$ between VD from the lower half of the retina and sum of sensitivities in the upper half of the visual fields and a weak correlation between RNFL SH and VF IH ( $\mathrm{r}=0.37)$, RNFL IH and VF $\mathrm{SH}(\mathrm{r}=0.32)$ respectively.

\section{DISCUSSION}

The effect of high IOP on retinal and choroidal vascularization was tried to prove experimentally by Patel et al. Because a significant portion of retinal thickness is comprised of vasculature, the purpose of the current study was to investigate OCT structural and vascular changes in healthy non-human primate eyes with systematic graded increases and decreases in IOP. They found that NOT does not have a direct effect on RNFL ( ref. $^{10}$ ).

Ma a et. all focused on changes in vessel density of the patients with narrow anterior chamber after an acute intraocular pressure elevation observed by OCT angiography. However, when the IOP rise was greater than $20 \mathrm{mmHg}$, the macular and papillary vessel density decreased significantly ${ }^{3}$.

Mansoori et al. investigated peripapillary VD in unilateral closed angle glaucoma (PACG). They found a significant difference in RNFL $(P<0.0001)$ and VD $(P=0.001)$ thickness between the upper and lower hemipoles. Within the perimetrically undamaged area, RNFL was significantly lower in the superior nasal and upper nasal sectors $(P=0.02)$ but VD showed no changes ${ }^{11}$. For NTG, a change in vascular density in the peripapillary region was registered $^{12-17}$.

The OAG and NTG groups evinced a lower superficial VD than did the control group, while the NTG group had a lower deep VD than the control group. The NTG group also had a larger foveal avascular zone than did the OHT group. No difference in choriocapillaris perfusion was observed among the groups ${ }^{18}$. The results of our research are in agreement with the above studies. By comparing RNFL and VD, we showed a medium correlation in HTG and NTG. By comparing VD from the upper half of the retina and the contralateral sum of sensitivities in the visual field, we showed a weak correlation in HTG and a strong correlation in NTG. We did not show any dependence in HTG when comparing VD from the lower half of the retina and the sum of sensitivity in the upper half of the visual fields. For NTG, this dependence was moderate. Our findings indicate that other than peripapillary vascular changes are more involved in functional changes in the visual fields of HTG, at least in the early stages of glaucoma, in contrast to NTG, where vascular changes play a crucial role in changes of visual field. We have not found a similar work as this in the literature. Even these conclusions allow us to conclude that HTG is a different diagnostic group than NTG. 
Table 1. Summary of the measured values in HTG group.

\begin{tabular}{|c|c|c|c|c|c|c|c|c|c|c|c|c|c|}
\hline \multirow[t]{2}{*}{ Sex-age } & \multicolumn{2}{|c|}{$\begin{array}{l}\text { RNFL RE } \\
{[\mu \mathrm{m}]}\end{array}$} & \multicolumn{2}{|c|}{$\begin{array}{l}\text { RNFL LE } \\
\quad[\mu \mathrm{m}]\end{array}$} & \multicolumn{2}{|c|}{ VD RE } & \multicolumn{2}{|c|}{ VD LE } & \multicolumn{2}{|c|}{$\begin{array}{l}\text { VF RE } \\
\text { [asb] }\end{array}$} & \multicolumn{2}{|c|}{$\begin{array}{l}\text { VF LE } \\
\text { [asb] }\end{array}$} & \multirow[t]{2}{*}{ Therapy } \\
\hline & $\mathrm{SH}$ & $\mathrm{IH}$ & $\mathrm{SH}$ & $\mathrm{IH}$ & SH & $\mathrm{IH}$ & SH & $\mathrm{IH}$ & $\mathrm{SH}$ & $\mathrm{IH}$ & SH & $\mathrm{IH}$ & \\
\hline F-1956 & 98 & 95 & 111 & 102 & 50 & 47 & 56 & 55 & 916 & 1041 & 1099 & 1046 & xalacom \\
\hline F-1948 & 88 & 97 & 79 & 81 & 47 & 44 & 46 & 47 & 1116 & 1073 & 1056 & 1061 & carteolol \\
\hline F-1962 & 98 & 101 & 110 & 101 & 59 & 59 & 56 & 54 & 1108 & 1104 & 1102 & 1067 & xalatan \\
\hline F-1950 & 113 & 109 & 114 & 104 & 49 & 49 & 49 & 52 & 1068 & 1072 & 1018 & 1003 & xalatan \\
\hline F-1943 & 89 & 102 & 102 & 98 & 52 & 52 & 56 & 53 & 963 & 1018 & 992 & 933 & timolol \\
\hline F-1959 & 104 & 102 & 103 & 103 & 52 & 50 & 48 & 47 & 1057 & 1053 & 1025 & 1015 & xalatan \\
\hline F-1958 & 116 & 104 & 108 & 98 & 54 & 51 & 50 & 47 & 1059 & 1058 & 1066 & 1037 & vizibim \\
\hline F-1965 & 87 & 76 & 82 & 85 & 45 & 42 & 46 & 41 & 1073 & 1043 & 1064 & 1030 & xalatan \\
\hline F-1944 & 98 & 103 & 101 & 102 & 48 & 46 & 46 & 43 & 1022 & 1020 & 984 & 986 & timolol \\
\hline F-1954 & 89 & 74 & 89 & 98 & 51 & 46 & 54 & 56 & 1032 & 1038 & 966 & 999 & xalacom \\
\hline F-1944 & 102 & 104 & 107 & 110 & 50 & 53 & 48 & 49 & 940 & 985 & 932 & 916 & xalatan \\
\hline F-1954 & 98 & 101 & 94 & 97 & 49 & 52 & 52 & 53 & 1093 & 1069 & 1060 & 1062 & vizibim \\
\hline F-1956 & 106 & 102 & 112 & 101 & 52 & 50 & 53 & 49 & 1052 & 1068 & 1070 & 1058 & duotrav \\
\hline F-1958 & 96 & 98 & 102 & 94 & 50 & 49 & 50 & 48 & 1060 & 1049 & 1069 & 1036 & monopost \\
\hline F-1947 & 118 & 113 & 117 & 112 & 57 & 52 & 58 & 54 & 1079 & 1047 & 970 & 1003 & xalatan \\
\hline M-1946 & 100 & 100 & 91 & 93 & 47 & 50 & 43 & 41 & 1064 & 1013 & 949 & 901 & carteolol \\
\hline M-1959 & 104 & 102 & 103 & 95 & 53 & 53 & 54 & 54 & 1048 & 1087 & 986 & 1040 & monopost \\
\hline M-1966 & 121 & 129 & 121 & 129 & 52 & 54 & 53 & 53 & 1037 & 1033 & 953 & 978 & ganfort \\
\hline M-1962 & 99 & 103 & 95 & 94 & 51 & 48 & 51 & 51 & 1057 & 1061 & 1056 & 1065 & xalacom \\
\hline M-1958 & 92 & 103 & 121 & 139 & 51 & 50 & 53 & 55 & 1030 & 988 & 1062 & 1005 & $\begin{array}{l}\text { monopost } \\
\text { dualkopt }\end{array}$ \\
\hline
\end{tabular}

RNFL - retinal nerve fibre layer, VD - vessel density, VF - visual field, RE - right eye, LE - left eye, SH - superior hemifield, IH - inferior hemifield

Table 2. Summary of measured values in NTG group.

\begin{tabular}{|c|c|c|c|c|c|c|c|c|c|c|c|c|c|}
\hline \multirow[t]{2}{*}{ Sex-age } & \multicolumn{2}{|c|}{$\begin{array}{l}\text { RNFL RE } \\
\quad[\mu \mathrm{m}]\end{array}$} & \multicolumn{2}{|c|}{$\begin{array}{l}\text { RNFL LE } \\
{[\mu \mathrm{m}]}\end{array}$} & \multicolumn{2}{|c|}{ VD RE } & \multicolumn{2}{|c|}{ VD LE } & \multicolumn{2}{|c|}{$\begin{array}{l}\text { VF RE } \\
\text { [asb] }\end{array}$} & \multicolumn{2}{|c|}{$\begin{array}{l}\text { VF LE } \\
\text { [asb] }\end{array}$} & \multirow[t]{2}{*}{ Therapy } \\
\hline & $\mathrm{SH}$ & $\mathrm{IH}$ & $\mathrm{SH}$ & $\mathrm{IH}$ & $\mathrm{SH}$ & IH & $\mathrm{SH}$ & $\mathrm{IH}$ & SH & IH & SH & IH & \\
\hline F-1956 & 92 & 87 & 90 & 87 & 48 & 45 & 49 & 48 & 1104 & 1092 & 982 & 1035 & 0 \\
\hline F-1949 & 127 & 123 & 124 & 128 & 52 & 53 & 50 & 51 & 1042 & 991 & 968 & 900 & latanoprost \\
\hline F-1954 & 96 & 104 & 92 & 100 & 50 & 51 & 49 & 52 & 1083 & 1012 & 1043 & 1012 & brinzolamid \\
\hline F-1955 & 98 & 101 & 92 & 99 & 51 & 51 & 49 & 48 & 1088 & 1148 & 1029 & 1074 & 0 \\
\hline F-1946 & 75 & 67 & 77 & 94 & 27 & 28 & 31 & 41 & 683 & 745 & 294 & 632 & brinzolamid \\
\hline F-1968 & 95 & 98 & 93 & 97 & 48 & 48 & 53 & 56 & 1186 & 1204 & 1152 & 1136 & 0 \\
\hline F-1953 & 109 & 107 & 107 & 103 & 51 & 48 & 51 & 50 & 1042 & 1074 & 1092 & 1077 & betoptic \\
\hline F-1944 & 112 & 94 & 105 & 104 & 54 & 53 & 53 & 50 & 1099 & 1068 & 968 & 1026 & 0 \\
\hline F-1956 & 89 & 97 & 94 & 83 & 46 & 46 & 49 & 45 & 1060 & 1055 & 1015 & 1001 & latanoprost \\
\hline F-1946 & 88 & 88 & 53 & 81 & 51 & 53 & 40 & 50 & 1035 & 990 & 977 & 880 & brimonidin \\
\hline F-1953 & 103 & 122 & 103 & 110 & 52 & 53 & 50 & 52 & 1104 & 1073 & 1115 & 1106 & carteolol \\
\hline F-1940 & 93 & 93 & 106 & 99 & 41 & 39 & 47 & 43 & 955 & 940 & 942 & 921 & timolol \\
\hline F-1960 & 89 & 90 & 93 & 61 & 48 & 51 & 49 & 42 & 977 & 999 & 755 & 944 & brimonidin \\
\hline F-1966 & 96 & 100 & 104 & 97 & 51 & 52 & 52 & 52 & 1073 & 952 & 1074 & 1038 & brimonidin \\
\hline F-1948 & 118 & 114 & 121 & 124 & 56 & 57 & 54 & 54 & 909 & 1023 & 1030 & 973 & latanoprost \\
\hline F-1976 & 112 & 112 & 114 & 111 & 51 & 50 & 45 & 47 & 1098 & 1093 & 1073 & 1089 & 0 \\
\hline F-1960 & 93 & 90 & 97 & 104 & 52 & 52 & 53 & 53 & 1092 & 1095 & 1084 & 1105 & carteolol \\
\hline M-1956 & 76 & 78 & 91 & 82 & 41 & 39 & 48 & 47 & 1093 & 907 & 1013 & 1013 & brimonidin \\
\hline M-1968 & 89 & 86 & 88 & 93 & 48 & 48 & 51 & 52 & 925 & 960 & 990 & 881 & betoptic \\
\hline M-1953 & 80 & 96 & 102 & 110 & 48 & 47 & 50 & 49 & 1038 & 1063 & 1035 & 1079 & betoptic \\
\hline
\end{tabular}

RNFL - retinal nerve fibre layer, VD - vessel density, VF - visual field, RE - right eye, LE - left eye, SH - superior hemifield, IH - inferior hemifield 
Table 3. Pearson's correlation coefficients in HTG and NTG group.

\begin{tabular}{|c|c|c|c|c|c|c|}
\hline DATA & VD SH & VF IH & VF IH & VD IH & VF SH & VF SH \\
\hline GROUP & RNFL SH & RNFL SH & VD SH & RNFL IH & RNFL IH & VD IH \\
\hline HTG & 0.50 & -0.04 & 0.26 & 0.51 & -0.12 & 0.04 \\
\hline NTG & 0.62 & 0.37 & 0.70 & 0.59 & 0.32 & 0.52 \\
\hline
\end{tabular}

$r=0.00-0.19$ very weak, $0.20-0.39$ weak, $0.40-0.59$ moderate, $0.60-0.79$ strong, $0.80-1.00$ very strong

\section{CONCLUSSION}

The results show a difference between the two diagnostic groups. NTG will probably be an ischemic lesion, which may not play such a role in HTG.

\section{ABBREVIATIONS}

HTG, hypertensive glaucoma; NTG, normotensive glaucoma; IOP, intraocular pressure; VD, vessel density; OCTA, optical coherence tomography angiography; POAG, primary open angle glaucoma; asb, apostilb; $\mathrm{SH}$, superior hemifield; IH, inferior hemifield; OCT, optical coherent tomography; RNFL, retinal nerve fibre layer; MRW, minimum rim width; BMO, Bruch's membrane opening; ALCS, anterior lamina cribrosa surface; PAGC, primary angle closure glaucoma; GCC, ganglion cell complex; VF, visual field.

Authors contribution: MF, JL, KM: manuscript writing, final approval; KM, JL: literature search, data analysis. Conflict of interest statement: The authors state that there are no conflicts of interest regarding the publication of this article.

\section{REFERENCES}

1. Shou T Liu J, Wang W, Zhou Y, Zhao K. Differential dendritic shrinkage of alpha and beta retinal ganglion cells in cats with chronic glaucoma. Invest Ophthalmol Vis Sci 2003;44:3005-10.

2. Mammo Z, Heisler M, Balaratnasingam C, Lee S, Yu DY, Mackenzie P, Schendel S, Merkur A, Kirker A, Albiani D, Navajas E, Beg MF, Morgan W, Sarunic MV. Quantitative Optical Coherence Tomography Angiography of Radial Peripapillary Capillaries in Glaucoma, Glaucoma Suspect, and Normal Eyes. Am J Ophthalmol 2016;170:41 9.

3. Ma ZW, Qiu WH, Zhou DN, Yang WH, Pan XF, Chen H. Changes in vessel density of the patients with narrow antenior chamber after an acute intraocular pressure elevation observed by OCT angiography. BMC Ophthalmol 2019;19:132. doi: 10.1186/s12886-019-1146-6

4. Mangouritsas G, Koutropoulou N, Ragkousis A, Boutouri E, Diagourtas A. Peripapillary Vessel Density In Unilateral Preperimetric Glaucoma. Clin Ophthalmol 2019;13:2511-9.

5. Lešták J, Nutterová E, Pitrová Š, Krejčová H, Bartošová L, Forgáčová V. High tension versus normal tension glaucoma. A comparison of structural and functional examinations. J Clinic Exp Ophthalmol 2012;S:5. doi: 10.4172/2155-9570.S5-006
6. Maresova K, Lestak J, Fus M, Weissova I. Effect of prostaglandins and beta blockers on progression of hypertensive and normotensive glaucomas. Biomed Pap Med Fac Univ Palacky Olomouc Czech Repub 2020; Mar 26. doi: 10.5507/bp.2020.011. [Epub ahead of print] doi: $10.5507 /$ bp. 2020.011

7. Dastiridou A, Chopra V. Potential applications of optical coherence tomography angiography in glaucoma. Curr Opin Ophthalmol 2018;29:226-33.

8. Richter GM, Sylvester B, Chu Z, Burkemper B, Madi I, Chang R, Reznik A, Varma R, Wang RK. Peripapillary microvasculature in the retinal nerve fibre layer in glaucoma by optical coherence tomography angiography: focal structural and functional correlations and diagnostic performance. Clin Ophthalmol 2018;12:2285-96.

9. Lešták J, Fůs M, Benda A, Marešová K. OCT angiografie a dopplerovská sonografie u normotenzního glaukomu. Cesk Slov Oftalmol 2020;76:118-21.

10. Patel N, McAllister F, Pardon L, Harwerth R. The effects of graded intraocular pressure challenge on the optic nerve head. Exp Eye Res 2019;169:79-90.

11. Mansoori T, Balakrishna N. Peripapillary Vessel Density and Retinal Nerve Fiber Layer Thickness in Patients with Unilateral Primary Angle Closure Glaucoma with Superior Hemifield Defect. J Curr Glaucoma Pract 2019;13:21-7.

12. Scripsema NK, Garcia PM, Bavier RD, Chui TY, Krawitz BD, Mo S, Agemy SA, Xu L, Lin YB, Panarelli JF, Sidoti PA, Tsai JC, Rosen RB. Optical Coherence Tomography Angiography Analysis of Perfused Peripapillary Capillaries in Primary OpenAngle Glaucoma and Normal-Tension Glaucoma. Invest Ophthalmol Vis Sci 2016;57:611-20.

13. Igarashi $R$, Ochiai S, Sakaue $Y$, Suetake A, likawa R, Togano T, Miyamoto F, Miyamoto D, Fukuchi T. Optical coherence tomography angiography of the peripapillary capillaries in primary open-angle and normal-tension glaucoma. PLoS One 2017;15:12(9):e0184301. doi: 10.1371/journal.pone.0184301

14. Takeyama A, Ishida K, Anraku A, Ishida M, Tomita G. Comparison of Optical Coherence Tomography Angiography and Laser Speckle Flowgraphy for the Diagnosis of Normal-Tension Glaucoma. J Ophthalmol 31;2018:1751857. doi: 10.1155/2018/1751857

15. Tepelus TC, Song S, Borrelli E, Nittala MG, Baghdasaryan E, Sadda SR, Chopra V .Quantitative Analysis of Retinal and Choroidal Vascular Parameters in Patients With Low Tension Glaucoma. J Glaucoma 2019;28:557-62.

16. Kim JS, Kim YK, Baek SU, Ha A, Kim YW, Jeoung JW, Park KH3. Topographic correlation between macular superficial microvessel density and ganglion cell-inner plexiform layer thickness in glaucoma-suspect and early normal-tension glaucoma. Br J Ophthalmol 2020;104:104-9.

17. Nitta K, Sugiyama K, Wajima R, Tachibana G, Yamada Y. Associations between changes in radial peripapillary capillaries and occurrence of disc hemorrhage in normal-tension glaucoma. Graefes Arch Clin Exp Ophthalmol 2019;9:1963-70.

18. Chao SC, Yang SJ, Chen HC, Sun CC, Liu CH, Lee CY. Early Macular Angiography among Patients with Glaucoma, Ocular Hypertension, and Normal Subjects. J Ophthalmol 2019;15:7419470. doi: $10.1155 / 2019 / 7419470$ 